\title{
LA RACIONALIZACIÓN POST-TAYLORISTA Y LAS INVESTIGACIONES DE TRABAJO EN LA SOCIOLOGÍA ALEMANA DEL TRABAJO DESDE LA MITAD DE LA DÉCADA DE LOS NOVENTA
}

\author{
Otfried Mickler \\ Universidad de Hannover \\ E-mail: omickle@gwdg.de
}

\begin{abstract}
RESUMEN
La sociología de trabajo alemana nos revela el cambio que ocurre en la actualidad en la organización de la industria alemana bajo las principales ideas de producción enjuta. En lo que concierne a los trabajadores de fábrica, la existencia de una fuerte desigualdad en la distribución de carácterísticas importantes de las situaciones de trabajo se hace patente. Aunque notables grupos de empleados pueden considerarse como ganadores de la reestructuración, para la mayoría de trabajadores no especializados, las condiciones de trabajo poco favorables que soportan no han cambiado en gran medida. A pesar de que se podría lograr una mejoría en las condiciones de trabajo a través del trabajo en grupo organizado entre ellos mismos, dicha mejoría no podría extenderse sin un fuerte compromiso político por parte de las empresas y de los representantes sindicales. Aparentemente, y en la actualidad, este tipo de avance se encuentra únicamente en un número muy reducido de empresas. Esto se debe a la fuerte competencia experimentada en el mercado mundial, que da lugar a presiones de altos costes en las empresas, además de afectar las tasas de desempleo ya altas y debilitar en gran medida la fuerza en el negocio respecto de los empleados y sus representantes sindicales.
\end{abstract}

Durante los años ochenta, la organización taylorista de la industria ya había alcanzado sus límites, la racionalización taylorista en las fábricas había agotado sus posibilidades para incrementar la producción. La sociología del trabajo de Alemania Occidental había discutido la crisis del taylorismo precozmente, había analizado los conceptos nuevos emergentes de la racionalización en la industria y había investigado sus consecuencias sobre el trabajo indus- 
trial. Había un acuerdo generalizado en torno a la observación de que las empresas estaban probando estrategias post-tayloristas de racionalización, pero seguía poco clara la dirección en la que se dirigían los nuevos conceptos y cómo cambiarían las condiciones de trabajo de los empleados. A principios de los años noventa, el debate de la producción Lean, por la manera en la que se discutió por las empresas mismas y por la manera en que influyó en su reestructuración, determinó de forma crucial los temas y la perspectiva analítica de la actual investigación sociológica del trabajo. Quedó claro que las empresas líderes se encaminaban por una vía de reestructuración post-taylorista que no dependía únicamente de una mayor automatización de la fabricación, sino de un cambio general en la organización de la empresa. En las investigaciones empíricas, los sociólogos recogían e intentaban explicar de modo teórico los nuevos conceptos de la organización tales como la descentralización, la división del trabajo entre las empresas y el trabajo en equipo, conceptos que algunas empresas empezaban a poner en práctica. Además, ponían en duda, de forma crítica, si las condiciones de trabajo de los empleados realmente mejoraban a largo plazo, tal como anunciaban los profetas de la producción Lean (Womack et al., 1991).

En las secciones que siguen presentaré cuatro estudios empíricos de los años noventa que investigan la relación entre la racionalización post-taylorista y el trabajo desde perspectivas distintas: Schumann et al. (1994) se centran en la reorganización de los sectores automatizados de la industria, Deiß et al. analizan las redes de las empresas industriales, Mickler $e$ t al. investigan la transformación de la producción socialista a la producción Lean en Alemania Oriental (1996) y Gerst et al. (1997) examinan el trabajo en grupo en la sala de producción. Estos estudios se basan en estudios intensivos de casos en fábricas que se apoyan en las entrevistas expertas con los directores y los consejos de trabajo; además, tres de los cuatro estudios emplean la observación de los lugares de trabajo y las entrevistas con los trabajadores.

\section{TRENDREPORT RATIONALISIERUNG}

El estudio Trendreport Rationalisierung (Schumann et al., 1994), planificado como el seguimiento del Ende der Arbeitsteilung? de Kern/Schumann (1984), investigó dos cuestiones: ¿ Realmente ganan aceptación en la industria los «conceptos nuevos de producción», tal como Kern y Schumann pronosticaron en 1984? ¿Cómo cambian con las nuevas estrategias de racionalización las estructuras del trabajo? Diseñado de forma muy amplia, la encuesta empírica duró desde 1986 hasta 1992 e incluyó a 12 fábricas de la industria automovilística, 4 empresas de la industria química y farmacéutica y 10 de la ingeniería mecánica. Utilizando una mezcla de varios métodos, los científicos de SOFIGöttingen llevaron a cabo 96 observaciones de lugares de trabajo, 300 entrevistas con trabajadores y 500 entrevistas de expertos. Además, estudiaron los 
datos estructurales cuantitativos de numerosos empleos — de 79.200 empleos en la industria automovilística, 12.000 en la industria química y 4.400 en la ingeniería mecánica- (Schumann et al., 1994: 38).

Los resultados del estudio confirman el pronóstico de Kern y Schumann. Durante los años ochenta, nuevos conceptos de la organización y nuevas formas cualificadas de trabajo se extendieron de hecho, paulatinamente dentro de los sectores de alta tecnología de las fábricas. La dirección siguió automatizando de manera extensa estas áreas, pero a la vez se dió cuenta de que el trabajo humano cualificado es necesario para la operación de los sistemas técnicos complejos. Las empresas están probando nuevos conceptos de organización que dependen sobre todo del «regulador de sistemas», un tipo nuevo de trabajo de automatización.

Los reguladores de sistemas son todos los trabajadores que tienen que vigilar el proceso automatizado y que no tienen que hacer trabajo manual de forma permanente. La programación de ordenadores, el control de los procesos, la localización de averías y el mantenimiento son las tareas más importantes, además de las tareas ocasionales sencillas como el control de calidad, la limpieza o la alimentación manual de las máquinas. El regulador de sistema es responsable de asegurar el alto rendimiento de un proceso automatizado continuo. El trabajo de regulación de sistemas puede organizarse a varios niveles de cualificación, según la variedad de tareas y las intervenciones en el proceso que se requieren. Los autores descubrieron varios conceptos de organización en las empresas, que diferían según el número de los niveles verticales y horizontales de la división del trabajo. Éstos abarcan desde un incremento en la división del trabajo, pasando por formas de integración limitada de las funciones, hasta un alto nivel de integración que comprende el mantenimiento y el control de calidad. Así, Schumann et al. (1994) encontraron una tendencia hacia el empleo de trabajadores cualificados, típicamente en equipos de doble función con antiguos trabajadores de mantenimiento.

La tarea y el grado de rendimiento del regulador de sistemas son fundamentalmente nuevos, desde que en el trabajo de automatización las tareas están desligadas del proceso productivo. No tienen ya que manipular materiales en la fabricación repetitiva, sino asegurar el funcionamiento de una tecnología de producción compleja e inestable. Las intervenciones necesarias para resolver los problemas que surjan no pueden anticiparse totalmente y, por lo tanto, el trabajo exige la capacidad de autoorganización. Sin embargo, las empresas no reaccionaron adecuadamente a esta necesidad. Llevaron a la práctica una integración horizontal de tareas sin ninguna regulación formal que permitiera a los reguladores de sistemas organizar sus propios grupos. No se estableció el trabajo en grupo autoorganizado en la tradición de los "grupos autónomos».

Los conceptos nuevos de producción casi siempre llevaron a mejoras en la situación de trabajo, como demostraron los análisis de trabajo y las entrevistas a los trabajadores de Schumann y sus colegas. Los reguladores de sistema encontraron mucho más atractivo su trabajo después de la integración de las 
tareas. Tienen mayor capacidad discrecional y una situación de trabajo más interesante sin la necesidad de aceptar un estrés adicional de rendimiento. Sin embargo, sería equivocado suponer que las condiciones del trabajo de regulación de sistema armonizan completamente los intereses de los trabajadores y de la empresa. Mientras que los conflictos tradicionales relacionados con la intensificación del trabajo perdieron relevancia, asuntos relacionados con las condiciones del trabajo tales como el ruido y el trabajo en turnos mantuvieron su importancia. Sorprendentemente, una nueva lista de exigencias surgió: los reguladores de sistemas exigieron integración de tareas y autonomía de trabajo adicionales; querían aún más competencias y afirmaron que las jerarquías existentes ya no satisfacían los requisitos nuevos de la producción de alta tecnología.

Tomando como ejemplo la industria automovilística, ya que puede considerarse que ésta marca el paso de la reestructuración industrial en Alemania, los reguladores de sistemas constituían aproximadamente el 8 por 100 de todos los trabajadores de producción, tal como Schumann et al. demostraron (ibid., S. 66ff.). Por lo tanto, se puede argumentar que los «nuevos conceptos de producción» apenas afectaban a los sectores de baja tecnología. Las áreas del trabajo manual en la industria automovilística seguían estructuradas de forma taylorista hasta los años noventa. Poco cambió durante este período para más del 90 por 100 del trabajo en la fabricación de coches, siendo éste de forma predominante de operadores de máquinas poco cualificados y de trabajadores de cadenas de montaje. Aunque ocurrieron algunos cambios aislados en la organización del trabajo, las soluciones solían ser bastante convencionales. La dirección se dio parcialmente cuenta de los fallos del trabajo taylorista, especialmente en la cadena de montaje, pero no se comprometieron en abordar una transformación fundamental hacia las nuevas formas de organización. Los autores observaron sólo algunas alteraciones pequeñas y aisladas: se eliminaron parcialmente las cadenas de montaje con el uso de la nueva tecnología de transporte, se expandieron de modo cauteloso los ciclos de trabajo, se hicieron algunas mejoras ergonómicas, se introdujeron grupos de trabajo con rotaciones internas para trabajos de ciclo corto, y se integraron el manejo de material y el control de calidad (ibid., S. 266ff.; Kurz, 1998). En las áreas de cadena de montaje, aún dominantes, el cambio organizacional se limitó a la integración de tareas sencillas de control de calidad y a una mayor flexibilidad a través de la rotación de trabajos.

El Trendreport Rationalisierung pone de manifiesto que estos cambios menores no afectaron al entendimiento tradicional de los trabajadores del trabajo y del rendimiento. Mientras que los reguladores de sistema enfatizaron en su percepción del trabajo dimensiones de la motivación intrínseca como la responsabilidad y la autonomía, los trabajadores manuales expresaron una concienciación más tradicional del trabajo. Seguían poniendo énfasis sobre las normas cuantitativas del rendimiento y las condiciones en las que había que cumplir con este rendimiento. Los autores llegaron a la conclusión de que las 
políticas de desarrollo del trabajo en los sectores de baja tecnología en la industria automovilística alemana no eran adecuadas para cambiar la naturaleza del trabajo restrictivo y enajenado.

Más que ningún otro estudio sociológico de los últimos años, el Trendreport Rationalisierung ha actualizado y ha creado una base empírica amplia y comprobada para el conocimiento de las estructuras de trabajo y los perfiles de capacitación de los trabajadores industriales. Aunque Schumann et al. se centraron en el trabajo en la sala de producción, en esta área analizaron a fondo el desarrollo de la tecnología y de la organización de las fábricas, a través de lo cual explicaron los cambios observados en el trabajo en las áreas de alta y de baja tecnología. Subrayaron la importancia crítica de los conceptos organizacionales de las empresas para el diseño del trabajo; según estos autores, el trabajo de producción no se puede mejorar sustancialmente ni se puede reprofesionalizar con sólo la automatización, sino únicamente con la innovación organizacional. Entienden que ésta es la razón detrás de la tendencia hacia una mejora significativa del trabajo en las áreas de alta tecnología, que varía entre las distintas empresas. Muchas empresas vuelven hacia un paradigma taylorista, porque la automatización permite mayor margen para una organización cualificada del trabajo. Pero, aun dentro de esta tendencia, las empresas se deciden a favor de conceptos alternativos de la organización y, de acuerdo con esta decisión, escogen maneras distintas de dividir el trabajo. Por esto, los investigadores encontraron el tipo de trabajo del "regulador de sistemas» en una gama variable de niveles de capacitación. Por lo tanto, subrayan la importancia decisiva de la política de trabajo de la empresa para la mejora del trabajo industrial.

\section{LA DIVISIÓN DEL TRABAJO Y LA RACIONALIZACIÓN SISTEMÁTICA ENTRE LAS EMPRESAS}

El estudio «Las nuevas estrategias de la racionalización y la racionalización entre las empresas», del ISF-Munich, investigó un rasgo característico de la racionalización industrial al cual, durante mucho tiempo, no se había prestado atención: la tendencia a construir redes entre las empresas y a reconfigurar la división del trabajo entre las empresas. La investigación comenzó en 1987 en la industria mobiliaria, pero pronto se centró completamente en la industria automovilística, incluidas sus numerosas empresas de suministro. Los resultados se publicaron en varias antologías y en algunos ensayos (Altmann/Sauer, 1989; Deiß/Döhl, 1992; Deiß, 1994). Los autores interpretaron las redes novedosas entre las grandes empresas de fabricación y de suministro, según el acercamiento desarrollado por el ISF-Munich de "racionalización sistémica»*, y examinaron las siguientes preguntas: ¿Hasta qué punto se desarrollan las nuevas formas de racionalización en conexión con la construcción de redes

* Cp. O. Mickler, Sociology of Labour in Germany 1999, capítulo 3: en el mismo tomo. 
entre las empresas? ¿Se están sustituyendo las relaciones de mercado actuales entre las empresas por otros mecanismos de coordinación? Como resultado de esto, ¿cómo cambian las estructuras de poder y las relaciones de dependencia entre las empresas? ¿De qué manera influye esta forma de racionalización sistémica en las condiciones de trabajo de los empleados en las empresas de fabricación y de suministro?

$\mathrm{Al}$ escoger la industria automovilística, los investigadores del ISF eligieron un campo de investigación en el cual la construcción de redes entre las empresas ha sido especialmente llamativa desde finales de los años ochenta. Para aumentar la flexibilidad y la productividad de las empresas, las corporaciones automovilísticas desarrollaron una forma nueva de producción masiva flexible en la cual se asignó un papel importante a las empresas de suministro (Deiß, 1994: 427). Así, las empresas de compras, siendo las empresas capaces de planificar y fabricar dentro de las corporaciones automovilísticas, están construyendo una red de empresas de suministro legalmente independientes, que sin embargo, están estrechamente vinculadas con la empresa industrial a través de su logística, y cuyo flujo de fabricación se está controlando detalladamente a través de la tecnología de la información. En la trayectoria de esta reorganización, la gran empresa industrial reduce su propia producción al agotar las fuentes de algunos componentes complejos, incluidas las funciones asociadas de diseño y desarrollo. A la vez intenta recoger los aumentos en productividad resultantes para sí mismo, poniendo precios objetivo para los suministradores e imponiendo por la fuerza unas reducciones regulares de precio. Estas redes ampliamente ramificadas en forma de pirámide han surgido desde las empresas de compras, pasando por los suministradores de sistemas, que desarrollan y producen los componentes complejos, hasta los subcontratistas, que producen piezas sencillas, todos los cuales muestran distintos grados de dependencia, según su posición en la pirámide. Se está desarrollando un nuevo equilibrio entre la autonomía y el control entre las empresas de compradores y las de suministros. Mientras se está desmontando la jerarquía en la gran empresa industrial y se están descentralizando las decisiones al abrir la organización a los principios de mercado, el grado de supervisión y de control jerárquico a través de la red sobre las empresas de suministros está aumentando. Nuevas estructuras de poder están surgiendo, en las que la empresa industrial domina y en las que la dependencia de los suministradores aumenta (ibid.: 427).

Dentro de las redes de producción, los investigadores del ISF observaron una escisión entre las empresas dominantes, es decir, las empresas de compradores y los suministradores de sistemas, y las empresas que son comparativamente más dependientes, es decir, la mayoría de los subcontratistas, que son básicamente pequeños. Los subcontratistas, especialmente, están sometidos a la presión sobre los precios por las empresas automovilísticas y están amenazados de forma existencial por la competencia internacional de la búsqueda global de fuentes. De acuerdo con esto, tal como mostraron los investigadores, se está desarrollando dentro de la red una fuerte segmentación de los empleados, en 
cuanto a condiciones de trabajo y de sueldo. Como las empresas de compradores y los suministradores de sistemas desplazan a los subcontratistas los requisitos de flexibilidad inducidos por el mercado, los riesgos ocupacionales y las condiciones desfavorables de trabajo se acumulan de modo predominante allí. Debido a la alta presión sobre los costes, estos subcontratistas están racionalizando sus empresas de forma continua y están reduciendo plantilla, están aumentando la flexibilidad de la producción y están intensificando el rendimiento. Los investigadores encontraron, en las empresas de suministradores de sistemas, sistemas automatizados y reguladores de sistemas cualificados, en relación con los nuevos conceptos de organización, pero sus vínculos estrechos con las empresas de compradores, especialmente en cuanto a las producciones "justo a tiempo», causan una presión intensa de tiempo y una flexibilidad extremada en el uso de la mano de obra. Por el contrario, la fabricación en los subcontratistas está marcada básicamente por el taylorismo: la flexibilidad necesaria se alcanza con la rotación de trabajos en las tareas sencillas, se emplea con preferencia mano de obra barata, y se socavan las normas sociales de los acuerdos salariales (ibid.: 432).

Especialmente para los trabajadores de los subcontratistas, esto resulta en altas tensiones y en una situación de empleo en conjunto inseguro. Según la situación de pedidos, los trabajadores tienen que alternar entre horas reducidas de trabajo, horas extras y turnos de noche; se traslada a los trabajadores a puestos distintos dentro de la fábrica; y varían de forma extremada los requisitos para los puestos. Debido a los estrechos vínculos de información y tecnológicos con la empresa de compradores, se vuelve transparente el rendimiento del suministrador: se les pide a los trabajadores que racionalicen su propio trabajo con Kaizen. Están bajo un estrés permanente, que amenaza su salud (ibid.: 437).

Como conjunto, este estudio del ISF-Munich va más allá de la sala de fabricación misma, en el campo tradicional de la investigación en la sociología industrial alemana. Ante la racionalización sistémica con redes ampliamente ramificadas, la sociología industrial no puede limitarse ya a los procesos sencillos de fabricación o a una empresa en solitario. Como argumentan los autores, es necesario investigar la división del trabajo entre las empresas. Además, este estudio contribuye sustancialmente a la reflexión sociológica sobre el debate acerca de la producción Lean en la industria, indicando que las redes piramidales de suministro no son, como se sugiere en la literatura de dirección, una cooperación armoniosa de dar y tomar en condiciones de igualdad, sino que incorporan relaciones de dependencia asimétricas.

\section{UN MODELO POSTFORDISTA DE ORGANIZACIÓN EN ALEMANIA ORIENTAL}

Con la agitación que acompañó al cambio desde el socialismo estatal a la economía de mercado, el proyecto de investigación «La modernización de la 
industria automovilística en Alemania Oriental» (Mickler et al., 1996) se encontró con la rara oportunidad de investigar la construcción de nuevas fábricas y de nuevas redes regionales entre las nuevas empresas según el modelo de los trasplantes japoneses. Se plantearon las siguientes preguntas: ¿Hasta qué punto tienen éxito las empresas automovilísticas al introducir un concepto organizacional tan exigente como la producción Lean en una industria socialista completamente anticuada? Habiendo sido formados en el propio trabajo en las fábricas socialistas estatales, ¿cómo se las arreglan los trabajadores de Alemania Oriental con los nuevos requisitos? Este estudio se basa en 12 casos de estudio de empresas, que constan de 75 entrevistas con expertos, de 200 entrevistas con trabajadores y empleados y de la inspección de lugares de trabajo.

El estudio trata de la transformación, en muchos sentidos difícil, de la antigua industria de Alemania Oriental en una economía de mercado. Se cuestionaba no sólo la transformación del anterior sistema político y económico, sino además la tarea crítica de la modernización comprensiva de la sociedad industrial de Alemania Oriental. La industria automovilística de Alemania Oriental fue un buen ejemplo de este proceso radical de transformación, ya que era una industria especialmente anticuada pero ahora, en su forma renova$\mathrm{da}$, tiene buenas posibilidades de sobrevivir. Con inversiones masivas en este área, las compañías occidentales (Volkswagen y General Motors/Opel) han puesto en práctica un tipo nuevo de innovación organizacional según el modelo de la producción Lean.

Así, los inversores han creado nuevas redes regionales entre las empresas en Alemania Oriental. Éstas constan de pocas empresas de fabricación y muchas de suministro tipo "justo a tiempo», que ganan aproximadamente el 70 por 100 del total del valor añadido generado localmente. También es de notar la descentralización e integración avanzadas de las tareas de dirección y de los servicios internos: las empresas son de tamaño medio, la dirección coopera de manera estrecha e informal y la distancia entre los niveles altos y bajos de dirección es mínima. Esto permite una comunicación mucho menos burocrática que en las empresas enormes y altamente burocratizadas del Occidente, acelera las decisiones y disminuye los gastos generales.

La dirección, además, ha dado forma a la organización del trabajo en la sala de producción según los principios de la producción Lean: en las empresas de fabricación de VW-Mosel y Opel-Eisenach se ha introducido el trabajo en grupo. Sin embargo, este trabajo en grupo se parece más a los modelos conocidos de los trasplantes japoneses en los Estados Unidos que al trabajo en grupo autónomo de Suecia (f.e. Uddevalla), como se demuestra en particular en el ejemplo de Opel-Eisenach. Las personas trabajan en grupos pequeños, turnándose dentro del grupo, pero siguen atadas a los ciclos cortos de la cadena de montaje - no se enriquece el trabajo con funciones de servicio cualificadas-. El grupo se supone que se autodirige, pero no tiene mucho margen para hacerlo. Se le pide que aumente la eficacia de la fabricación con el Kaizen, pero el líder del grupo, elegido por la dirección, domina esta optimización inteligente 
de la fabricación. A causa de la política de asignar escasos recursos humanos a los grupos, se les anima a seguir aumentando la productividad con el Kaizen. Sin embargo, esto aumenta la presión para rendir, lo cual da como resultado unas condiciones bastante desfavorables para un proceso de aprendizaje del grupo (Mickler et al., 1996: 111).

De esta manera han surgido fábricas de coches altamente eficientes, contándose entre las más productivas de Europa. Las empresas automovilísticas incluso las ponen como fábricas modelo para la reorganización de las fábricas antiguas de Alemania Occidental. Puesto que hay una fuerte competencia internacional para la asignación de los pedidos de fabricación y de las inversiones dentro de las corporaciones, las antiguas fábricas de Alemania Occidental tienen que competir con las altamente eficientes fábricas Lean de Alemania Oriental. Esto acelera la reorganización de las grandes empresas de Alemania Occidental y, a la vez, presiona a los empleados y a los consejos de trabajo para que acepten objetivos de rendimiento tan duros y de contratación de trabajadores tan estrictos como en Alemania Oriental. Así, la modernización de la industria automovilística de Alemania Oriental entra dentro de la política de globalización de las empresas automovilísticas como una estrategia importante.

Con este trasfondo, los investigadores estudiaban la cuestión de cómo se las arreglaban los trabajadores y empleados de Alemania Oriental con las demandas de una fábrica Lean, siendo ésta una tarea completamente nueva para ellos. Los cambios dramáticos en las empresas automovilísticas han implicado nuevos requisitos y nuevas orientaciones de trabajo para la mayoría de los empleados que seguían. Para cumplir con los altos estándares de calidad de productos, el nuevo modelo postfordista de organización requiere un alto nivel de motivación de los trabajadores manuales, para trabajar duro y de forma continua todos los días y para cooperar con los jefes de los niveles más bajos. Esto significaba que los trabajadores tenían que desarrollar un comportamiento completamente nuevo que era, de alguna manera, contrario al comportamiento que habían aprendido anteriormente en las fábricas socialistas. Al mirar retrospectivamente este proceso de modernización, se puede llegar a la conclusión de que tanto los trabajadores de cuello azul como los de cuello blanco de las nuevas fábricas automovilísticas de Alemania Oriental finalmente han podido hacer frente a estos nuevos requisitos. Según los investigadores, esto se debió tanto al potencial tradicional de los trabajadores de Alemania Oriental como a las condiciones específicas del proceso de transformación.

Desde luego, un factor importante es el personal joven y cualificado que los gestores reclutaron de entre un gran número disponible de trabajadores cualificados después del colapso de la economía de la República Democrática Alemana. Los trabajadores están sobrecualificados para las tareas básicamente sencillas de las fábricas y tienen un potencial alto de aprendizaje. En estas circunstancias, normalmente se esperaría una alta insatisfacción con el contenido del trabajo y, de acuerdo con esto, una baja motivación para trabajar entre los trabajadores cualificados. De hecho, una gran proporción de los trabajadores 
se quejan de la monotonía del trabajo actual, pero la insatisfacción permanece latente y no desemboca en una reducción del rendimiento. Esto probablemente se debe a varias causas. Las entrevistas muestran que los trabajadores tienen expectativas muy modestas acerca de las demandas en el contenido del trabajo y que están preparados para aguantar el sufrimiento. En los tiempos de la República Democrática Alemana estaban acostumbrados a aceptar condiciones malas de trabajo sin quejarse; era parte de su adaptación a los defectos de la sociedad RDA. Hoy día, con las inseguridades derivadas de las transformaciones, ellos mismos se están retirando hacia la virtud de la modestia.

Sin embargo, una influencia crucial en el comportamiento laboral de los trabajadores, acostumbrados a décadas de una seguridad absoluta de empleo, es sobre todo el desempleo extremadamente alto de Alemania Oriental. Con esta experiencia masiva de la inseguridad social y el temor acerca del trabajo propio, un trabajo no cualificado y poco exigente les parece, en comparación, un problema menor, aparentando ser así de poca importancia. Comparada con las alternativas inexistentes, la posibilidad de trabajar en tareas restrictivas, pero en fábricas automovilísticas que dan seguridad para el futuro, no les parece una mala alternativa.

Utilizando el ejemplo del levantamiento de las fábricas Lean, el estudio de Mickler et al. permite penetrar en los conceptos postfordistas de organización, apenas investigados hasta ahora, aunque sea en una industria que se ha modernizado en condiciones sociales especiales. La rápida revolución del socialismo de estado a la economía de mercado y la globalización de la industria automovilística caracterizaron de manera crítica la dinámica y la forma de la reestructuración como dos mecanismos interconectados. Con el ejemplo de las empresas de Alemania Oriental, los procesos de aprendizaje organizacional para hacer frente a la reestructuración de conjunto de las fábricas se podían estudiar, y se podían analizar las consecuencias sociales para los empleados. Se encontró, de acuerdo con los conocimientos de la sociología organizacional, que las buenas condiciones para hacer frente con éxito a una reestructuración difícil son: poca distancia jerárquica, una comunicación densa, el consenso y una capacitación buena y homogénea de los empleados. Sin embargo, al contrario de la ideología de la producción Lean, los investigadores observaron formas bastante poco favorables de trabajo para los trabajadores en la sala de producción. Esto lo explicaron a través del marco general que es específico a Alemania Oriental: por ejemplo, un mercado de trabajo extremadamente desfavorable, consejos de trabajo débiles y la muy buena disposición de los empleados a adaptarse, los cuales permitieron a los directivos poner en práctica una organización bastante estrecha del trabajo, dejando a los trabajadores en la sala de producción con poca decisión discrecional y poca participación. 


\section{EL TRABAJO EN GRUPO EN LA SALA DE PRODUCCIÓN}

Los investigadores del SOFI-Göttingen (Gerst et al., 1998; Schumann et al., 1997) estudiaron el trabajo en grupo en la industria automovilística, donde se ha difundido rápidamente desde 1993. Se preguntaron: ¿Qué condiciones y constelaciones políticas dentro de las empresas apoyan el desarrollo del trabajo en grupo? ¿Qué formas de trabajo en grupo se pueden distinguir empíricamente y cuáles son las consecuencias del trabajo en grupo sobre los trabajadores? El estudio se enfocó en seis fábricas de dos empresas automovilísticas alemanas, Mercedes Benz y Volkswagen, y en una gran empresa de suministros, con la investigación de aproximadamente 80 grupos. Se utilizó una mezcla de métodos, compuesta de la observación intensiva de lugares de trabajo y de grupos, entrevistas de expertos con directivos y consejos de trabajo, y entrevistas abiertas y estandarizadas con trabajadores.

Ya en los años setenta, apoyada en el programa de los gobiernos sobre la calidad de la vida en el trabajo, había habido una discusión vívida en la industria alemana acerca del rediseño del trabajo con la ayuda del trabajo en grupo, conceptos orientados por el acercamiento sociotécnico de las empresas suecas. Pero sólo se habían llevado a la práctica los cambios en las salas de producción de modo muy restringido hasta principios de los años, debido a la resistencia interna. La discusión de la producción Lean y la presión de la crisis en la industria automovilística han llevado a una rápida difusión del trabajo en grupo en las salas de producción desde 1993. Para la dirección, los conceptos de equipo se convirtieron en el principio central de la organización moderna de las fábricas para conseguir incrementar la productividad y para lograr mayor flexibilidad. Durante los últimos años, la mayor parte de las empresas han estado experimentando con conceptos como los del trabajo en grupo, la descentralización y Kaizen. Por lo tanto, la realidad de la sala de producción se caracteriza ahora por una gran variedad de formas de trabajo en grupo. El término "trabajo en grupo» se utiliza de manera inflacionaria y vaga en la discusión científica: a menudo se clasifica de modo indiscriminado como trabajo en grupo tanto el trabajo restrictivo como el cualificado, organizado en grupos. Así, Gerst et al. proponen que se distingan dos líneas básicas de desarrollo, colocadas en extremos opuestos de los dos polos del trabajo en grupo. Uno de los modelos, inspirado por los trasplantes japoneses, difiere en sólo unos pocos aspectos del concepto taylorista de organización. Se puede llamar «trabajo en grupo taylorista modificado». Este modelo se ha perfilado en el capítulo 3 en la descripción de la fábrica Lean de Opel-Eisenach en Alemania Oriental. El otro modelo de los conceptos de grupo contemporáneos, que a menudo se llama autoorganizador o trabajo en grupo autónomo, se caracteriza por una diferencia fundamental comparado con las estructuras tayloristas previas en la fabricación. Gerst et al. representan los elementos clave de este modelo de trabajo en grupo autoorganizado de la siguiente manera (Gerst et al., 1998: 5 ss.): 
- Tanto el diseño de los trabajos como el poder discrecional son extendidos. Las funciones de servicio se incluyen con el propósito de aumentar la variedad de las tareas y las destrezas en el trabajo.

- La autoorganización y la capacidad de decisión de los grupos son los objetivos principales del desarrollo. Para conseguir esto se proporcionan los recursos apropiados: se eligen vocales del grupo, quienes siguen formando parte del grupo a la vez que actúan como vocales y coordinadores. Reuniones autoorganizadas de grupo ayudan a mejorar la coordinación y a solucionar problemas tanto de fabricación como de comunicación.

- Este concepto de trabajo en grupo requiere un papel nuevo de la dirección de bajo nivel en la sala de producción. Se debe delegar en los grupos la responsabilidad y las funciones del servicio. Los directivos de los niveles más bajos no deben intervenir como un superior, sino actuar como un entrenador.

Este concepto también toma en serio la participación en el lugar de trabajo. El grupo mismo se incluye en las negociaciones de los salarios del trabajo. Además, está involucrado en el desarrollo de todo el proceso de producción, lo cual da como resultado una mejora en las condiciones de trabajo del grupo.

Además, Gerst et al. informan que Mercedes empezó a introducir el trabajo en grupo que corresponde al concepto de autoorganización a principios de los años noventa. El mismo proceso se pudo observar en Volkswagen, tal como pone de manifiesto una investigación reciente de Mickler et al. (1998). El concepto de trabajo en grupo autoorganizado parece coincidir con rasgos institucionales alemanes tales como el trabajo cualificado de producción, la codeterminación y la política de los sindicatos (trabajadores del metal - IG Metall) dirigidos hacia el trabajo en grupo cualificado. Las empresas Mercedes y Volkswagen intentaron formar estrategias a nivel de toda la corporación a través de la negociación de acuerdos con los consejos de trabajo, que incluían las características del trabajo en grupo autoorganizado, los recursos necesarios y el modo de introducir este trabajo en grupo. Sin embargo, a pesar de este concepto dominante para toda la empresa, aún hay un gran margen de discreción dentro de la fábrica para la puesta en práctica del trabajo en grupo. En gran medida, los cambios en la sala de producción dependen de las políticas de los actores respectivos y del proceso social de implementación, por ejemplo, de las políticas de la dirección local y de los consejos de trabajo. Éstos incluso tienen autoridad para modificar el acuerdo general si existe un consenso entre ellos dentro de la fábrica.

Como demuestran las investigaciones de Gerst et al. y de Mickler et al., la puesta en práctica del trabajo en grupo comenzó de manera prometedora y dinámica en ambas empresas. Se dotó generosamente a los proyectos de grupo de recursos y plantilla adicional, se les dio tiempo para discusiones de grupo y posibilidades de formación. Sin embargo, los distintos grupos se desarrollaron de modo muy diferente, especialmente si se comparan las áreas de producción de alta y de baja tecnología. 
Los casos más positivos de desarrollo de grupo los encontraron Gerst et al. en las áreas de alta tecnología; por ejemplo, en el caso de una fábrica de piezas de motor en la cual los operadores de las máquinas tenían que fabricar tubos de escape en ciclos cortos y en remesas grandes (Schumann et al., 1997, S. 136 ss.). Después de la reestructuración, el trabajo dentro de los grupos se caracteriza por una integración bastante amplia de tareas, incluidas las funciones de servicio: la rotación da a todo el mundo la oportunidad de aprender a hacer cada tarea. Como resultado, han surgido perfiles de trabajo enriquecidos, uniformes y solapados, mostrando una mejora que permite una alta flexibilidad en el uso del trabajo. Además, los trabajadores también han desarrollado una autoorganización de largo alcance de su trabajo. Las tareas diarias de los grupos incluyen la organización de la asignación de trabajadores a las máquinas, la planificación de las vacantes y la secuencia en la que se despacharán los pedidos. La autoorganización se apoya en vocales de grupo elegidos, quienes están integrados al grupo y tienen que llevar a cabo trabajo de producción — su papel se puede describir como de coordinador, moderador y vocal del grupo-. Sin embargo, el grupo entero toma las decisiones, no el vocal solo. Una vez a la semana se asigna tiempo de discusión para los grupos, que los trabajadores utilizan para solucionar problemas de producción y sociales.

En las áreas de baja tecnología, especialmente en las áreas de cadena de montaje, Gerst et al. también encontraron algunos casos de grupos autoorganizados, pero prevalecían de forma aplastante las variaciones restrictivas de trabajo en grupo. Había, por ejemplo, un caso de trabajo en grupo situado en el área de recortes de la última cadena de montaje: aquí el trabajo en grupo se limitaba fundamentalmente a la fusión de varias tareas en un ciclo de trabajo más largo (Gerst et al., 1998, S. 16). La rotación de trabajos está muy restringida: la mayoría de los miembros del grupo sólo están capacitados para llevar a cabo unos pocos trabajos debido a una falta de oportunidades de formación. De acuerdo con esto, la flexibilidad entre los colegas es baja. El grupo también muestra una pequeña medida de autoorganización. El vocal de grupo está en una posición especial. No modera ni representa al grupo hacia fuera, sino que actúa exclusivamente como un especialista con responsabilidades específicas como la reelaboración o la adquisición de piezas. Aunque los líderes del grupo fueron elegidos, los directivos de los niveles más bajos (Meister) les consideraban sustitutos de los antiguos capataces y les presionaban para que cumpliesen los objetivos impuestos por la dirección. En la práctica, ha evolucionado un modelo que en muchos aspectos se parece al trabajo de grupo taylorista perfilado antes: la naturaleza del trabajo en grupo está fuertemente influenciada por las estructuras tayloristas que perduran.

Para concluir, Gerst et al. dicen que, en resumen, su investigación de esta reestructuración pone de manifiesto que la puesta en práctica de conceptos notayloristas de la organización es un proceso social que es altamente dependiente de un conjunto de premisas. Son necesarias las políticas de dirección con varios niveles jerárquicos y una codirección activa que consiste en representan- 
tes laborales; sin estos elementos no se puede alcanzar el ímpetu para un cambio radical de la organización (Gerst et al., 1998). El concepto del grupo de trabajo autoorganizado está respaldado por los trabajadores y lo ven como una mejora fundamental de sus condiciones de trabajo. La mejora de las capacitaciones y la expansión de las responsabilidades estimulan el compromiso de los trabajadores en la solución de problemas y proporcionan una base para la participación. Esto se aplica en particular a las áreas de alta tecnología. Por otra parte, en las áreas de baja tecnología, por ejemplo las cadenas de montaje, un incremento de la productividad por conceptos de grupo da como resultado un incremento en la carga de trabajo. Sin embargo, los trabajadores de cadena de montaje en grupos autoorganizados son más capaces de controlar estas presiones, debido a su ámbito de acción común. Los trabajadores, en cambio, dentro de los conceptos modificados de grupo taylorista, no son capaces de hacer frente a la intensificación del trabajo a través de la cooperación, es decir, esta forma de trabajo en grupo restrictivo no mejora la situación negativa de los trabajadores de las cadenas de montaje.

Aunque el trabajo en grupo se está introduciendo de forma amplia dentro de la industria automovilística alemana, las condiciones de trabajo de los trabajadores en las áreas de baja tecnología, con mucho la mayoría de los empleados, no están mejorando de modo sustancial. Estos resultados de Gerst et al. están en la misma línea que los del Trendreport Rationalisierung (Schumann et al., 1994). No permiten predicciones optimistas para el desarrollo futuro del trabajo de baja tecnología, especialmente considerando que las empresas automovilísticas en Alemania, desde hace unos años, han empezado a revisar su concepto actual de organización. Las empresas ya no se interesan mucho por los conceptos de grupos autoorganizados, y están cada vez más volviendo a la estandarización del trabajo de la cadena de montaje, acortando los ciclos y utilizando como modelo los conceptos japoneses de grupo. Tal cambio de concepto fue observado por Schumann et al. en Mercedes (Schumann et al., 1997: 160). Un desarrollo similar se puede ver en Volkswagen, donde la dirección ya descentralizada está recortando los recursos de los grupos para reducir costes, pero a la vez está estrechando el ámbito de acción de los grupos de forma extremada. En muchos casos, el trabajo en grupo está regresando a los conceptos tayloristas modificados (Mickler et al., 1998: 273).

\section{CONCLUSIÓN}

En conjunto, los cuatro estudios que se han relatado de la sociología industrial profundizan con una visión detallada en los cambios actuales de la organización en la industria alemana (con el ejemplo predominante de la industria automovilística), cambios que están teniendo lugar en la fábrica y en la sala de producción desde principios de los años noventa con las ideas directrices de la producción Lean. La reestructuración de las grandes empresas ha 
resultado ser un proceso extensivo y altamente dinámico, que sólo se ha investigado e interpretado sociológicamente en parte, aun después de la publicación de los estudios citados. En lo que concierne a los trabajadores de la sala de producción, y la mayoría de los resultados tratan este tema, emerge con claridad la existencia de una distribución notablemente desigual de las características importantes de las situaciones de trabajo. A diferencia del mensaje predicado por los profetas de la producción Lean (Womack et al., 1991), en cuanto a la mayoría de los trabajadores de producción, no se puede de ninguna manera hablar de una capacidad de decisión, de una mejora y una mayor capacidad discrecional en el lugar de trabajo. Aunque grupos notables de empleados (por ejemplo, en las áreas de alta tecnología) pueden considerarse ganadores en la reestructuración, para la mayoría de los trabajadores de baja tecnología, el trabajo en grupo no ha efectuado grandes cambios en sus desfavorables condiciones de trabajo. Aunque se podrían mejorar de modo sustancial sus condiciones de trabajo a través del trabajo en grupo autoorganizado, como demuestran los casos positivos dentro de las empresas, su difusión extensiva requeriría un fuerte compromiso político de las empresas y los representantes sindicales, que en la actualidad sólo se puede encontrar en muy pocas empresas. Por una parte, esto se debe a la fuerte competencia dentro del mercado mundial, que causa una alta presión sobre los costes en las empresas, y, por otra parte, al alto nivel de desempleo, que debilita de forma severa el poder de negociación de los empleados y sus representantes sindicales. Por lo tanto, la resistencia ante las variaciones desfavorables de la producción Lean, tales como los conceptos tayloristas modificados de trabajo de grupo, es particularmente débil en las áreas más fuertemente expuestas a la presión de un mercado de trabajo desfavorable: los trabajadores no-cualificados en las áreas de baja tecnología, los subcontratistas en las redes entre las empresas y los empleados en las regiones de niveles altos de desempleo, como en el caso de Alemania Oriental.

Al señalar la tendencia a la escisión, entre los trabajadores industriales, entre "ganadores» y "perdedores» como consecuencia de la reestructuración, los nuevos estudios vuelven a uno de los antiguos temas de la sociología industrial alemana. Ya en los años ochenta, Kern y Schumann llaman la atención sobre la segmentación de la fuerza de trabajo en ganadores y perdedores de la racionalización, y recomendaron la puesta en práctica de contramedidas con la ayuda de una política innovadora de trabajo (Kern/Schumann, 1984). En los nuevos estudios se repite este consejo con una similitud sorprendente, a pesar de que en la actualidad se enfoque más a los trabajadores en las áreas de baja tecnología que en aquel momento. 


\title{
BIBLIOGRAFÍA
}

Altmann, N., y Sauer, D. (1989): Systemische Rationalisierung und Zulieferindustrie, Frankfurt/New York.

DEIß, M. (1994): Arbeit in der Autozulieferindustrie, WSI-Mitteilungen, Heft 7.

DeIß, M., y KÖHL, V. (1992): Vernetzte Produktion, Frankfurt/New York.

Gerst, D.; Hardwig, T.; Kuhlmann, M., y Schumann, M. (1998): "Group Work in the German Automobile Industry», en J. P. Durand, P. Stewart y J. J. Castillo (eds.), Teamwork in the Automobile Industry, Macmillan Press.

KuRZ, C. (1999): Repetitivarbeit-unbewältigt, Berlin.

Mickler, O.; Engelhard, N.; Lungwitz, R., y Walker, B. (1996): Nach der Trabi-Ära Arbeiten in schlanken Fabriken, Berlin.

Mickler, O.; Hollmann, R., y Niemeyer, E. (1998): Gestaltungsansätze und soziale Prozesse, Univeröffentl, Manuskript, Hannover.

Schumann, M.; Baethge-Kinsky, V.; Kuhlmann, M.; KurZ, C., y Neumann, U. (1994): Trendreport Rationalisierung, Berlin.

Schumann, M., y Gerst, D. (1997): «Produktionsarbeit - Bleiben die Entwidklungstrends stabil?», en Jahrbuch sozialwissenschaftliche Technikberichterstattung 1996, Berlin.

Womack, J. P.; Jones, D. T., y Roos, D. (1991): Die zweite Revolution in der autoindustrie, Frankfurt/New York.

\begin{abstract}
German sociology of labour gives insight into the present changing of organisation in German industry, that is taking place under the leading ideas of ean production. As the workers on the shop-floor are concerned, the existence of a strikingy unequal distribution of important characteristics of the working situations becomes clear. Although noticeable groups of employees can be considered winners of restructuring, for the majority of low-tech workers group work has not changed their unfavourable working conditions much. Although working conditions could be substantially improved through self-organised group-work, its extensive spread would require a strong political commitment by companies and labour representatives. A developement like this can at present apparently be found only in very few companies. This is due to stiff competition within the world market, causing high cost pressure to the companies; high unemployment also severely weakens the bargaining power of employees and their labour representatives.
\end{abstract}




\section{TEXTO CLÁSICO}

